\title{
Note on identities inspired by new soft theorems
}

\author{
Junjie $\mathbf{R a o}^{a}$ and Bo Feng ${ }^{a, b}$ \\ ${ }^{a}$ Zhejiang Institute of Modern Physics, Zhejiang University, \\ Hangzhou, 31002\%, P.R. China \\ ${ }^{b}$ Center of Mathematical Science, Zhejiang University, \\ Hangzhou, 310027, P.R. China \\ E-mail: raojunjie@zju.edu.cn, b.feng@cms.zju.edu.cn
}

\begin{abstract}
The new soft theorems, for both gravity and gauge amplitudes, have inspired a number of works, including the discovery of new identities related to amplitudes. In this note, we present the proof and discussion for two sets of identities. The first set includes an identity involving the half-soft function which had been used in the soft theorem for one-loop rational gravity amplitudes, and another simpler identity as its byproduct. The second set includes two identities involving the KLT momentum kernel, as the consistency conditions of the KLT relation plus soft theorems for both gravity and gauge amplitudes. We use the CHY formulation to prove the first identity, and transform the second one into a convenient form for future discussion.
\end{abstract}

KEYwords: Scattering Amplitudes, Gauge Symmetry

ArXiv ePrint: 1604.00650 


\section{Contents}

1 Introduction 1

2 Two identities of the half-soft function 3

2.1 A simpler byproduct identity 4

$\begin{array}{ll}2.2 & \text { Proof of the first identity }\end{array}$

3 Two identities of the KLT momentum kernel 9

$\begin{array}{ll}3.1 \text { Proof of the first identity } & 10\end{array}$

$\begin{array}{lll}3.2 & \text { Discussion of the second identity } & 12\end{array}$

\section{Introduction}

Scattering amplitudes often have an universal soft behavior when the momentum of one external leg tends to zero. This soft limit can be traced back to the works [1-6]. Recently, a new soft theorem for tree level gravity amplitudes was studied in [7]. By using the on-shell recursion relation $[8,9]$ and imposing the holomorphic soft limit, Cachazo and Strominger have proved that

$$
\begin{aligned}
& M_{n}\left(\lambda_{n} \rightarrow \varepsilon \lambda_{n}\right) \\
& \quad=\frac{1}{\varepsilon^{3}} \sum_{a=1}^{n-2} \frac{\langle n-1, a\rangle^{2}[n a]}{\langle n-1, n\rangle^{2}\langle n a\rangle} M_{n-1}\left(\tilde{\lambda}_{n-1} \rightarrow \tilde{\lambda}_{n-1}+\varepsilon \frac{\langle a n\rangle}{\langle a, n-1\rangle} \tilde{\lambda}_{n}, \tilde{\lambda}_{1} \rightarrow \tilde{\lambda}_{1}+\varepsilon \frac{\langle n-1, n\rangle}{\langle n-1, a\rangle} \tilde{\lambda}_{n}\right)+O\left(\varepsilon^{0}\right),
\end{aligned}
$$

here for $M_{n}$ and $M_{n-1}$, the unmentioned external kinematic data are un-deformed and we prefer to suppress them for conciseness. Taylor expansion in $\varepsilon$ exhibits three singular terms in orders $\varepsilon^{-3}, \varepsilon^{-2}$ and $\varepsilon^{-1}$, while higher order terms in $\varepsilon$ will be mixed with the less interesting $O\left(\varepsilon^{0}\right)$ parts.

A similar relation for tree level Yang-Mills amplitudes using the on-shell recursion relation, proved by Casali [10], takes the form

$$
\begin{aligned}
& A_{n}\left(\lambda_{n} \rightarrow \varepsilon \lambda_{n}\right) \\
& \quad=\frac{1}{\varepsilon^{2}} \frac{\langle n-1,1\rangle}{\langle n-1, n\rangle\langle n 1\rangle} A_{n-1}\left(\tilde{\lambda}_{n-1} \rightarrow \tilde{\lambda}_{n-1}+\varepsilon \frac{\langle 1 n\rangle}{\langle 1, n-1\rangle} \tilde{\lambda}_{n}, \tilde{\lambda}_{1} \rightarrow \tilde{\lambda}_{1}+\varepsilon \frac{\langle n-1, n\rangle}{\langle n-1,1\rangle} \tilde{\lambda}_{n}\right)+O\left(\varepsilon^{0}\right),
\end{aligned}
$$

where two singular terms in orders $\varepsilon^{-2}$ and $\varepsilon^{-1}$ appear after Taylor expansion. The mixing between higher order terms from the deformed $A_{n-1}$ and $O\left(\varepsilon^{0}\right)$ parts also persists to this case.

Based on this new discovery, many related studies have been done. In [23-36], the soft theorem has been generalized to arbitrary dimensions and other theories or categories: 
string theory, ABJM theory, theories with fermions or massive particles, and form factors. In $[37-51,53-58]$, the theorem has been understood from various perspectives, especially those of symmetries and invariance. In [11, 28, 59-63], its generalization to loop level has been discussed. In [64-71], the relevant double (or multiple) soft theorem has also been discussed.

Among these studies, we have met two sets of identities which have not been proved so far. We will present the proof in this note.

One identity of the first set was mentioned in [11], which explored loop correction to the soft theorem. It involves the so-called half-soft function $h$ (first defined in [12] and reinterpreted in [13]), which appears naturally for all-plus one-loop gravity amplitude. Its general proof was not given in [11], but explicit checks up to 12 points had been done. The identity reads

$$
\sum_{b \neq n}\langle b n\rangle^{2} \sum_{M, N} h(b, n, M) h(b, n, N)\left\langle b\left|K_{M}\right| n\right]\left\langle n\left|K_{N}\right| b\right]^{3}=0
$$

where $M, N$ are two nonempty partition sets of the $(n-2)$ particles other than $b$ and $n$, and $K_{M}$ and $K_{N}$ are the corresponding total momenta. During the proof, we had also discovered another simpler identity, which can serve as its logical preliminary. It reads

$$
\frac{[1 n]}{\langle 1 n\rangle} \frac{\left|\psi_{N \cup M}\right|_{w}^{w}}{\langle w 1\rangle\langle w n\rangle}+\sum_{N}\left\langle 1\left|K_{N}\right| n\right]\left\langle n\left|K_{N}\right| 1\right] \frac{\left|\psi_{N}\right|_{x}^{x}}{\langle x 1\rangle\langle x n\rangle} \frac{\left|\psi_{M}\right|_{y}^{y}}{\langle y 1\rangle\langle y n\rangle}=0
$$

where the $\psi$ matrix is related to $h$, and other symbols above will be explained shortly.

The second set of identities was conjectured in [14], which is a consequence of consistency conditions between the soft theorems for gravity and gauge amplitudes, under the well-known KLT relation [15]. It involves the KLT momentum kernel [12, 16-18], and the transformation matrices ( $D$ and $C$ below) between BCJ basis of gauge amplitudes [19]. These two identities are

$$
\begin{gathered}
\sum_{\alpha_{t^{\prime}}, \beta_{t^{\prime}} \in S_{n-3}} D\left[t, \alpha_{t}, n-1, n \mid t^{\prime}, \alpha_{t^{\prime}}, n-1, n\right] S\left[\alpha_{t^{\prime}} \mid \beta_{t^{\prime}}\right]_{p_{n-1}} \\
\cdot C\left[t^{\prime}, n-1, \beta_{t^{\prime}}, n \mid t, n-1, \beta_{t}, n\right]=S\left[\alpha_{t} \mid \beta_{t}\right]_{p_{n-1}}, \\
\sum_{t^{\prime}=1}^{n-2} \sum_{\alpha_{t^{\prime}}, \beta_{t^{\prime}} \in S_{n-3}} D\left[t, \alpha_{t}, n-1, n \mid t^{\prime}, \alpha_{t^{\prime}}, n-1, n\right] S\left[\alpha_{t^{\prime}} \mid \beta_{t^{\prime}}\right]_{p_{n-1}} \\
\cdot J_{t^{\prime}}\left(C\left[t^{\prime}, n-1, \beta_{t^{\prime}}, n \mid t, n-1, \beta_{t}, n\right]\right)=0,
\end{gathered}
$$

where $S\left[\alpha_{t} \mid \beta_{t}\right]_{p_{n-1}}$ is the KLT momentum kernel of pivot $p_{n-1}$, and $J_{t^{\prime}} \equiv J_{t^{\prime}, \dot{\alpha} \dot{\beta}}$ is the anti-holomorphic angular momentum operator. We will use the CHY formulation [20-22] to prove the first identity and discuss the second one.

This note is organized as follows. In section 2, we prove identity (1.3) of the half-soft function, and also the byproduct identity (1.4). In section 3, we prove identity (1.5) of the KLT momentum kernel by using the CHY formulation, while we transform identity (1.6) into a convenient form for possible future attempts and end with some discussion. 


\section{Two identities of the half-soft function}

In this section we will prove (1.3) and (1.4), first let's set up a bit convenient facilitation. For reader's reference, we write (1.3) again below

$$
\sum_{b \neq n}\langle b n\rangle^{2} \sum_{M, N} h(b, n, M) h(b, n, N)\left\langle b\left|K_{M}\right| n\right]\left\langle n\left|K_{N}\right| b\right]^{3}=0,
$$

where $M, N$ are two non-overlapping nonempty sets satisfying $M \cup N=\{1, \ldots, n-1\} \backslash b$, and momentum conservation enforces $k_{b}+k_{n}+K_{M}+K_{N}=0$. The half-soft function $h$ above is defined as [13]

$$
h(b, n, N)=\frac{1}{\prod_{i}^{N}\langle i b\rangle^{2}\langle i n\rangle^{2}}|\Psi|_{r}^{r}=\frac{1}{\prod_{i}^{N}\langle i b\rangle^{2}\langle i n\rangle^{2}}\|\Psi\|,
$$

where $|\Psi|_{r}^{r}$ denotes the determinant of matrix $\Psi$ after deleting its $r$-th row and $r$-th column, and $\|\Psi\|$ indicates this quantity is independent of the choice $r \in N$. If there is only one row and one column, the determinant is 1 after deletion. The matrix $\Psi$ is defined as

$$
\Psi_{i j}(b, n)=-\frac{[i j]}{\langle i j\rangle}\langle i b\rangle\langle i n\rangle\langle j b\rangle\langle j n\rangle \text { for } i \neq j, \Psi_{i i}=\sum_{j \neq i}^{N} \Psi_{i j},
$$

where $b$ and $n$ serve as auxiliary spinors. The sum of each row is zero, so $\Psi$ is degenerate. Observe that the summand in (1.3) has even power of $K_{M}$ and $K_{N}$, by momentum conservation this sum is symmetric between $M$ and $N$, then we can replace $K_{M}$ by $-K_{N}$ and rewrite (1.3) as

$$
\sum_{b \neq n}\langle b n\rangle^{2} \sum_{N} h(b, n, N) h(b, n, M)\langle b|N| n]\langle n|N| b]^{3}=0,
$$

for brevity $N$ stands for $K_{N}$ in spinorial products (and later $N$ also represents the number of elements in the set $N$, depending on the context).

To simplify the proof, we define the matrix $\psi$ as

$$
\psi_{i j}(b, n)=-\frac{[i j]}{\langle i j\rangle}\langle j b\rangle\langle j n\rangle \text { for } i \neq j, \psi_{i i}=\sum_{j \neq i}^{N} \psi_{i j},
$$

where the common factor $\langle i b\rangle\langle i n\rangle$ of the $i$-th row in $\Psi$ has been stripped off. One can easily verify that

$$
h(b, n, N)=\frac{1}{\prod_{i}^{N}\langle i b\rangle^{2}\langle i n\rangle^{2}}\|\Psi\|=\frac{1}{\prod_{i}^{N}\langle i b\rangle\langle i n\rangle} \frac{\left|\psi_{N}\right|_{x}^{x}}{\langle x b\rangle\langle x n\rangle},
$$

where $N$ has been added to $\psi$ to label the corresponding set, note that $\left|\psi_{N}\right|_{x}^{x} /\langle x b\rangle\langle x n\rangle$ is independent of the choice $x \in N$. Then we have

$$
\begin{aligned}
h(b, n, N) h(b, n, M) & =\frac{1}{\prod_{i}^{N+M}\langle i b\rangle\langle i n\rangle} \frac{\left|\psi_{N}\right|_{x}^{x}}{\langle x b\rangle\langle x n\rangle} \frac{\left|\psi_{M}\right|_{y}^{y}}{\langle y b\rangle\langle y n\rangle} \\
& =\frac{\langle b n\rangle}{\prod_{i \neq n}\langle i n\rangle \prod_{i}^{N+M}\langle i b\rangle} \frac{\left|\psi_{N}\right|_{x}^{x}}{\langle x b\rangle\langle x n\rangle} \frac{\left|\psi_{M}\right|_{y}^{y}}{\langle y b\rangle\langle y n\rangle},
\end{aligned}
$$


where $\prod_{i \neq n}\langle i n\rangle$ is a common factor independent of $b$ so it can be dropped, hence (2.4) becomes

$$
\sum_{b \neq n} \frac{\langle b n\rangle^{3}}{\prod_{i \neq b, n}\langle i b\rangle} \sum_{N}\langle b|N| n]\langle n|N| b]^{3} \frac{\left|\psi_{N}\right|_{x}^{x}}{\langle x b\rangle\langle x n\rangle} \frac{\left|\psi_{M}\right|_{y}^{y}}{\langle y b\rangle\langle y n\rangle}=0 .
$$

\subsection{A simpler byproduct identity}

In the proof of (2.8), we happened to discover (1.4). For reader's reference, it is given below

$$
\frac{[1 n]}{\langle 1 n\rangle} \frac{\left|\psi_{N \cup M}\right|_{w}^{w}}{\langle w 1\rangle\langle w n\rangle}+\sum_{N}\langle 1|N| n]\langle n|N| 1] \frac{\left|\psi_{N}\right|_{x}^{x}}{\langle x 1\rangle\langle x n\rangle} \frac{\left|\psi_{M}\right|_{y}^{y}}{\langle y 1\rangle\langle y n\rangle}=0,
$$

where $N, M$ are two non-overlapping nonempty sets satisfying $N \cup M=\{2, \ldots, n-1\}$, and the auxiliary spinors are 1 and $n$. Also note that $w \in N \cup M, x \in N, y \in M$ and it is free to switch the choices $w, x, y$ within each set. Since this is mandatory for (2.8) to hold, we will prove it first as the tricks used here are analogous to those for (2.8).

Now we will adopt the BCFW deformation and reduce it into an identity of the same form, but with one particle removed, in other words, we will perform an inductive proof. Before induction, the identity is confirmed analytically at lower points for $n=4,5,6$. For later convenience, we multiply it by a non-zero factor, yields

$$
\frac{1}{\prod_{i \neq 1, n}\langle i 1\rangle}\left(\frac{[1 n]}{\langle 1 n\rangle} \frac{\left|\psi_{N \cup M}\right|_{w}^{w}}{\langle w 1\rangle\langle w n\rangle}+\sum_{N}\langle 1|N| n]\langle n|N| 1] \frac{\left|\psi_{N}\right|_{x}^{x}}{\langle x 1\rangle\langle x n\rangle} \frac{\left|\psi_{M}\right|_{y}^{y}}{\langle y 1\rangle\langle y n\rangle}\right)=0,
$$

which is of course equivalent to (1.4). But now there are two advantages: the large $z$ behavior of its l.h.s. is improved, and it has the desired simple pole for residue evaluation, as we will soon see.

For generic $n$, consider BCFW deformation $\langle 1| n]$ and a particular pole $\langle 21\rangle$. Note that particles 1 and $n$ are special while the rest $(n-2)$ ones are symmetric, so it is sufficient to consider the residue of $\langle 21\rangle$ only, as all $\langle i 1\rangle$ 's with $i \in\{2, \ldots, n-1\}$ behave similarly. At $\langle 2 \hat{1}\rangle=0$, we have

$$
\left.\left.\left.|\hat{1}\rangle=|1\rangle-|n\rangle \frac{\langle 12\rangle}{\langle n 2\rangle}=|2\rangle \frac{\langle 1 n\rangle}{\langle 2 n\rangle}, \mid \hat{n}\right]=\mid n\right]+\mid 1\right] \frac{\langle 12\rangle}{\langle n 2\rangle}
$$

and

$$
|\hat{1}\rangle[1|+| 2\rangle[2|\equiv| 2\rangle\left[\hat{2} \mid, \quad\left[\hat{2} \mid=\left[2 \mid+\left[1 \mid \frac{\langle 1 n\rangle}{\langle 2 n\rangle},\right.\right.\right.\right.
$$

by which we mean to combine the momenta of particle $\hat{1}$ and 2 into that of particle $\hat{2}$, or more physically, particles $\hat{1}$ and 2 merge into particle $\hat{2}$. Including the deformed particle $\hat{n}$, the set $\{1,2, \ldots, n\}$ now shrinks into $\{\hat{2}, \ldots, \hat{n}\}$ while momentum conservation still holds, as what induction requires.

To locate pole $\langle 21\rangle$ in (2.10), we immediately find one in the overall factor. Naively, there might be another one under $\left|\psi_{N}\right|_{x}^{x}$ if we take $x=2$, for example. However, the expansion of $\left|\psi_{N}\right|_{x}^{x}$ in terms of $\langle 21\rangle$ will cancel this pole. In other words, $\left|\psi_{N}\right| \begin{aligned} & x \\ & x\end{aligned} /\langle x 1\rangle\langle x n\rangle$ is a polynomial of $\langle 21\rangle$ (one may also choose $x \neq 2$ to invalidate this pole), that's why the overall factor is mandatory. 
The next step is to analyze the large $z$ behavior of the l.h.s. in (2.10) before evaluating its residues at finite locations. To clarify the analysis, we further separate the second term in the parenthesis, and from now on we redefine $N, M$ to exclude particle 2 from them while $N^{\prime}, M^{\prime}$ denote the original sets. Depending on whether $N^{\prime}$ or $M^{\prime}$ contains particle 2, the set $\{2, \ldots, n-1\}$ has three types of splitting: $\{\{2\} \cup N, M\},\{N,\{2\} \cup M\}$ and $\{\{2\}, N \cup M\}$, where $N \cup M=\{3, \ldots, n-1\}$. So the second term becomes

$$
\begin{aligned}
& \sum_{N^{\prime}}\left\langle 1\left|N^{\prime}\right| n\right]\left\langle n\left|N^{\prime}\right| 1\right] \frac{\left|\psi_{N^{\prime}}\right|_{x}^{x}}{\langle x 1\rangle\langle x n\rangle} \frac{\left|\psi_{M^{\prime}}\right|_{y}^{y}}{\langle y 1\rangle\langle y n\rangle} \\
&=\sum_{\{2\} \cup N, M}(\langle 1|N| n]+\langle 12\rangle[2 n])(\langle n|N| 1]+\langle n 2\rangle[21]) \frac{\left|\psi_{N \cup\{2\}}\right|_{2}^{2}}{\langle 21\rangle\langle 2 n\rangle} \frac{\left|\psi_{M}\right|_{y}^{y}}{\langle y 1\rangle\langle y n\rangle} \\
& \quad+\sum_{N,\{2\} \cup M}\langle 1|N| n]\langle n|N| 1] \frac{\left|\psi_{N}\right|_{x}^{x}}{\langle x 1\rangle\langle x n\rangle} \frac{\left|\psi_{M \cup\{2\}}\right|_{2}^{2}}{\langle 21\rangle\langle 2 n\rangle}+[n 2][12] \frac{\left|\psi_{N \cup M}\right|_{w}^{w}}{\langle w 1\rangle\langle w n\rangle} .
\end{aligned}
$$

Also, the first term in (2.10) can be written as

$$
\frac{[1 n]}{\langle 1 n\rangle} \frac{\left|\psi_{N^{\prime} \cup M^{\prime}}\right|_{w}^{w}}{\langle w 1\rangle\langle w n\rangle}=\frac{[1 n]}{\langle 1 n\rangle} \frac{\left|\psi_{N \cup M \cup\{2\}}\right|_{2}^{2}}{\langle 21\rangle\langle 2 n\rangle} .
$$

Since the three $\psi$ 's in (2.14) and the first and second terms of (2.13) contain particle 2, we can choose to delete its corresponding row and column. Large $z$ power counting shows that all four terms in (2.13) and (2.14) behave as $z^{N+M-1}=z^{n-4}$ under $\left.\langle 1| n\right]$, but the overall factor in the front of (2.10) behaves as $z^{-(n-2)}$, which renders the entire expression as $z^{-2}$, so there is no boundary contribution. Therefore, via contour integration, the l.h.s. of (2.10) (denoted $I$ below) can be expressed as

$$
\oint_{z=0} \frac{d z}{z} I(z)=-\oint_{\langle 2 \hat{1}\rangle=0} \frac{d z}{z} I(z)-\ldots-\oint_{\langle n-1, \hat{1}\rangle=0} \frac{d z}{z} I(z)
$$

if the residue at $\langle 2 \hat{1}\rangle=0$ vanishes, by the symmetry among particles $\{2, \ldots, n-1\}$ the entire un-deformed expression must also vanish. Note the contribution from the overall factor in (2.10) is universal, so it can be dropped. At $\langle 2 \hat{1}\rangle=0$, after some algebra, the residue evaluation gives

$$
\frac{\left|\psi_{N \cup\{2\}}\right|_{2}^{2}}{\langle 21\rangle\langle 2 n\rangle} \rightarrow\left(\frac{\langle 1 n\rangle}{\langle 2 n\rangle}\right)^{N-1}(-\langle n|N| 2]) \frac{\left|\psi_{N}\right|_{x}^{x}}{\langle x 2\rangle\langle x n\rangle},
$$

recall that $\langle 21\rangle$ above is not a pole, while the real pole comes from the overall factor. Here $|\hat{1}\rangle$ is replaced by $|2\rangle$ up to a factor, after recalling (2.11). By expanding the determinant to the first order of $\langle 21\rangle$, then using the independence of choice $x$ to switch the deleted row and column for each term, we can collect a factor $(-\langle n|N| 2])$ as above. The similar (and simpler) story happens for

$$
\frac{\left|\psi_{M}\right|_{y}^{y}}{\langle y 1\rangle\langle y n\rangle} \rightarrow\left(\frac{\langle 1 n\rangle}{\langle 2 n\rangle}\right)^{M-2} \frac{\left|\psi_{M}\right|_{y}^{y}}{\langle y 2\rangle\langle y n\rangle} .
$$


Plugging them back, up to a factor $(\langle 1 n\rangle /\langle 2 n\rangle)^{N+M-2}$, the sum of (2.13) and (2.14) becomes

$$
\begin{aligned}
& (-\langle n|N+M| 2]) \frac{\left|\psi_{N \cup M}\right|_{w}^{w}}{\langle w 2\rangle\langle w n\rangle} \frac{[1 n]}{\langle 2 n\rangle} \\
& \quad+\sum_{N}\langle 2|N| \hat{n}](\langle n|N| 1]+\langle n 2\rangle[21])(-\langle n|N| 2]) \frac{\left|\psi_{N}\right|_{x}^{x}}{\langle x 2\rangle\langle x n\rangle} \frac{\left|\psi_{M}\right|_{y}^{y}}{\langle y 2\rangle\langle y n\rangle} \\
& \quad+\sum_{N}\langle 2|N| \hat{n}]\langle n|N| 1](-\langle n|M| 2]) \frac{\left|\psi_{N}\right|_{x}^{x}}{\langle x 2\rangle\langle x n\rangle} \frac{\left|\psi_{M}\right|_{y}^{y}}{\langle y 2\rangle\langle y n\rangle}+[\hat{n} 2][12] \frac{\left|\psi_{N \cup M}\right|_{w}^{w}}{\langle w 2\rangle\langle w n\rangle}
\end{aligned}
$$

By momentum conservation, up to a factor [12], it can be simplified into

$$
\begin{aligned}
& \left(\frac{\langle 1 n\rangle}{\langle 2 n\rangle}[n 1]+[\hat{n} 2]\right) \frac{\left|\psi_{N \cup M}\right|_{w}^{w}}{\langle w 2\rangle\langle w n\rangle} \\
& +\langle n 2\rangle \sum_{N}\langle 2|N| \hat{n}]\left(\langle n|N| 2]+\langle n|N| 1] \frac{\langle 1 n\rangle}{\langle 2 n\rangle}\right) \frac{\left|\psi_{N}\right|_{x}^{x}}{\langle x 2\rangle\langle x n\rangle} \frac{\left|\psi_{M}\right|_{y}^{y}}{\langle y 2\rangle\langle y n\rangle} \\
& \quad=\langle n 2\rangle\left(\frac{[\hat{2} \hat{n}]}{\langle 2 n\rangle} \frac{\left|\psi_{N \cup M}\right|_{w}^{w}}{\langle w 2\rangle\langle w n\rangle}+\sum_{N}\langle 2|N| \hat{n}]\langle n|N| \hat{2}] \frac{\left|\psi_{N}\right|_{x}^{x}}{\langle x 2\rangle\langle x n\rangle} \frac{\left|\psi_{M}\right|_{y}^{y}}{\langle y 2\rangle\langle y n\rangle}\right)=0
\end{aligned}
$$

after assuming the identity of $(n-1)$ particles holds. This finishes the inductive proof of (1.4).

\subsection{Proof of the first identity}

Now we move to prove (2.8) by applying the similar pack of tricks: to consider deformation $\langle 1| n]$ acting on its l.h.s., and the pole $\langle 21\rangle$. First, we separate the expression into three parts corresponding to $b=1, b=2$ and $b=3, \ldots, n-1$, namely

$$
\sum_{b \neq n} \frac{\langle b n\rangle^{3}}{\prod_{i \neq b, n}\langle i b\rangle} \sum_{N}\langle b|N| n]\langle n|N| b]^{3} \frac{\left|\psi_{N}\right|_{x}^{x}}{\langle x b\rangle\langle x n\rangle} \frac{\left|\psi_{M}\right|_{y}^{y}}{\langle y b\rangle\langle y n\rangle}=I_{1}+I_{2}+I_{b \neq 1,2, n} .
$$

Similarly, we now redefine $N$ and $M$ to exclude particles 2 and 1, with respect to $I_{1}$ and $I_{2}$. For $I_{1}$, the set $\{2, \ldots, n-1\}$ has three types of splitting: $\{\{2\} \cup N, M\},\{N,\{2\} \cup M\}$ and $\{\{2\}, N \cup M\}$, where $N \cup M=\{3, \ldots, n-1\}$. For $I_{2}$, we have $\{\{1\} \cup N, M\},\{N,\{1\} \cup M\}$ and $\{\{1\}, N \cup M\}$. For $I_{b \neq 1,2, n}$, there are four types: $\left\{\{1,2\} \cup N_{b}, M_{b}\right\},\left\{N_{b},\{1,2\} \cup M_{b}\right\}$, $\left\{\{1\} \cup N_{b},\{2\} \cup M_{b}\right\}$ and $\left\{\{2\} \cup N_{b},\{1\} \cup M_{b}\right\}$, where $N_{b} \cup M_{b}=\{3, \ldots, n-1\} \backslash b$, but the last two will not contribute to the residue of $\langle 21\rangle$ and hence the corresponding terms are neglected, which will be explained shortly.

According to the splittings above, we can write

$$
\begin{aligned}
\frac{I_{1}}{\langle 1 n\rangle^{3}}= & \frac{1}{\prod_{i \neq 1,2, n}\langle i 1\rangle\langle 21\rangle} \sum_{N}\left(\langle 1|N+2| n]\langle n|N+2| 1]^{3} \frac{\left|\psi_{N \cup\{2\}}\right|_{2}^{2}}{\langle 21\rangle\langle 2 n\rangle} \frac{\left|\psi_{M}\right|_{y}^{y}}{\langle y 1\rangle\langle y n\rangle}\right. \\
& \left.+\langle 1|N| n]\langle n|N| 1]^{3} \frac{\left|\psi_{N}\right|_{x}^{x}}{\langle x 1\rangle\langle x n\rangle} \frac{\left|\psi_{M \cup\{2\}}\right|_{2}^{2}}{\langle 21\rangle\langle 2 n\rangle}\right) \\
& +\frac{1}{\prod_{i \neq 1,2, n}\langle i 1\rangle\langle 21\rangle}\langle 1|2| n]\langle n|2| 1]^{3} \frac{1}{\langle 21\rangle\langle 2 n\rangle} \frac{\left|\psi_{N \cup M}\right|_{w}^{w}}{\langle w 1\rangle\langle w n\rangle},
\end{aligned}
$$




$$
\begin{aligned}
\frac{I_{2}}{\langle 2 n\rangle^{3}}= & \frac{1}{\prod_{i \neq 1,2, n}\langle i 2\rangle\langle 12\rangle} \sum_{N}\left(\langle 2|N+1| n]\langle n|N+1| 2]^{3} \frac{\left|\psi_{N \cup\{1\}}\right|_{1}^{1}}{\langle 12\rangle\langle 1 n\rangle} \frac{\left|\psi_{M}\right|_{y}^{y}}{\langle y 2\rangle\langle y n\rangle}\right. \\
& \left.+\langle 2|N| n]\langle n|N| 2]^{3} \frac{\left|\psi_{N}\right|_{x}^{x}}{\langle x 2\rangle\langle x n\rangle} \frac{\left|\psi_{M \cup\{1\}}\right|_{1}^{1}}{\langle 12\rangle\langle 1 n\rangle}\right) \\
& +\frac{1}{\prod_{i \neq 1,2, n}\langle i 2\rangle\langle 12\rangle}\langle 2|1| n]\langle n|1| 2]^{3} \frac{1}{\langle 12\rangle\langle 1 n\rangle} \frac{\left|\psi_{N \cup M}\right|_{w}^{w}}{\langle w 2\rangle\langle w n\rangle}, \\
I_{b \neq 1,2, n}= & \sum_{b \neq 1,2, n} \frac{\langle b n\rangle^{3}}{\prod_{i \neq 1, b, n}\langle i b\rangle\langle 1 b\rangle} \\
& \times \sum_{N_{b}}\left(\left\langle b\left|N_{b}+1+2\right| n\right]\left\langle n\left|N_{b}+1+2\right| b\right]^{3} \frac{\left|\psi_{N_{b} \cup\{1,2\}}\right|_{1}^{1}}{\langle 1 b\rangle\langle 1 n\rangle} \frac{\left|\psi_{M_{b}}\right|_{y}^{y}}{\langle y b\rangle\langle y n\rangle}\right. \\
& \left.+\left\langle b\left|N_{b}\right| n\right]\left\langle n\left|N_{b}\right| b\right]^{3} \frac{\left|\psi_{N_{b}}\right|_{x}^{x}}{\langle x b\rangle\langle x n\rangle} \frac{\left|\psi_{M_{b} \cup\{1,2\}}\right|_{1}^{1}}{\langle 1 b\rangle\langle 1 n\rangle}\right) \\
& + \text { (two neglected terms). }
\end{aligned}
$$

For $I_{b \neq 1,2, n}$ one can verify that, only terms for which 1 and 2 are in the same splitting set, have pole $\langle 21\rangle$ and hence contribute to the residue, which explains why we only need the first two terms. Moreover, $N_{b}$ in $\left\{\{1,2\} \cup N_{b}, M_{b}\right\}$ can be empty (similarly for $M_{b}$ ). While for $I_{1}, N$ in $\{\{2\} \cup N, M\}$ cannot be empty, otherwise such a splitting belongs to type $\{\{2\}, N \cup M\}$ (similarly for $I_{2}$ ).

After the separation, we now analyze the large $z$ behavior. Under $\langle 1| n]$, large $z$ power counting shows that $I_{1} \sim z^{-2}, I_{2} \sim z^{-1}$ and $I_{b \neq 1,2, n} \sim z^{-1}$, so there is no boundary contribution. Then we can repeat the contour integration (2.15). Again, thanks to the symmetry among particles $\{2, \ldots, n-1\}$, it is sufficient to consider the residue of $\langle 21\rangle$ only.

Recalling (2.16) and (2.17), at $\langle 2 \hat{1}\rangle=0$ the residue evaluation gives

$$
\begin{aligned}
\frac{\langle 21\rangle}{\langle 1 n\rangle^{3}} I_{1} \rightarrow & \frac{\langle 2 n\rangle^{3}}{\langle 1 n\rangle^{3}} \frac{1}{\prod_{i \neq 1,2, n}\langle i 2\rangle} \\
& \times \sum_{N}\left[\langle\hat{1}|N+2| \hat{n}]\langle n|N+2| 1]^{3}(-\langle n|N| 2])\right. \\
& \left.\quad+\langle\hat{1}|N| \hat{n}]\langle n|N| 1]^{3}(-\langle n|M| 2])\right] \frac{\left|\psi_{N}\right|_{x}^{x}}{\langle x 2\rangle\langle x n\rangle} \frac{\left|\psi_{M}\right|_{y}^{y}}{\langle y 2\rangle\langle y n\rangle} \\
& -\frac{\langle 2 n\rangle^{4}}{\langle 1 n\rangle^{2}} \frac{[12]^{3}[2 \hat{n}]}{\prod_{i \neq 1,2, n}\langle i 2\rangle} \frac{\left|\psi_{N \cup M}\right|_{w}^{w}}{\langle w 2\rangle\langle w n\rangle},
\end{aligned}
$$

or after a bit simplification,

$$
\begin{aligned}
\frac{\langle 21\rangle\langle 1 n\rangle}{\langle 2 n\rangle^{2}} I_{1} \rightarrow & \frac{\langle 1 n\rangle^{2}}{\prod_{i \neq 1,2, n}\langle i 2\rangle} \sum_{N}\langle 2|N| \hat{n}]( \\
& -\langle n|N| 2]\langle n|N+2| 1]^{3} \\
& \left.+\langle n|N+1| 2]\langle n|N| 1]^{3}\right) \frac{\left|\psi_{N}\right|_{x}^{x}}{\langle x 2\rangle\langle x n\rangle} \frac{\left|\psi_{M}\right|_{y}^{y}}{\langle y 2\rangle\langle y n\rangle} \\
& -\langle 1 n\rangle^{2}\langle 2 n\rangle^{2} \frac{[12]^{3}[2 \hat{n}]}{\prod_{i \neq 1,2, n}\langle i 2\rangle} \frac{\left|\psi_{N \cup M}\right|_{w}^{w}}{\langle w 2\rangle\langle w n\rangle}
\end{aligned}
$$


Similarly for $I_{2}$,

$$
\begin{aligned}
\frac{\langle 21\rangle\langle 1 n\rangle}{\langle 2 n\rangle^{2}} I_{2} \rightarrow & \frac{\langle 2 n\rangle^{2}}{\prod_{i \neq 1,2, n}\langle i 2\rangle} \sum_{N}\langle 2|N| \hat{n}]\left(\langle n|N| 1]\langle n|N+1| 2]^{3}\right. \\
& \left.-\langle n|N+2| 1]\langle n|N| 2]^{3}\right) \frac{\left|\psi_{N}\right|_{x}^{x}}{\langle x 2\rangle\langle x n\rangle} \frac{\left|\psi_{M}\right|_{y}^{y}}{\langle y 2\rangle\langle y n\rangle} \\
& -\langle 1 n\rangle^{3}\langle 2 n\rangle \frac{[12]^{3}[1 n]}{\prod_{i \neq 1,2, n}\langle i 2\rangle} \frac{\left|\psi_{N \cup M}\right|_{w}^{w}}{\langle w 2\rangle\langle w n\rangle} .
\end{aligned}
$$

Combining $I_{1}$ and $I_{2}$, we find

$$
\begin{aligned}
\frac{\langle 21\rangle\langle 1 n\rangle}{\langle 2 n\rangle^{2}}\left(I_{1}+I_{2}\right) \rightarrow & -\frac{[12]\langle 2 n\rangle^{3}}{\prod_{i \neq 1,2, n}\langle i 2\rangle} \sum_{N}\langle 2|N| \hat{n}]\langle n|N| \hat{2}]^{3} \frac{\left|\psi_{N}\right|_{x}^{x}}{\langle x 2\rangle\langle x n\rangle} \frac{\left|\psi_{M}\right|_{y}^{y}}{\langle y 2\rangle\langle y n\rangle} \\
& -\frac{[12]^{3}\langle 1 n\rangle^{2}\langle 2 n\rangle^{3}}{\prod_{i \neq 1,2, n}\langle i 2\rangle} \sum_{N}\langle 2|N| \hat{n}]\langle n|N| \hat{2}] \frac{\left|\psi_{N}\right|_{x}^{x}}{\langle x 2\rangle\langle x n\rangle} \frac{\left|\psi_{M}\right|_{y}^{y}}{\langle y 2\rangle\langle y n\rangle} \\
& -\langle 1 n\rangle^{2}\langle 2 n\rangle^{2} \frac{[12]^{3}[\hat{2} \hat{n}]}{\prod_{i \neq 1,2, n}\langle i 2\rangle} \frac{\left|\psi_{N \cup M}\right|_{w}^{w}}{\langle w 2\rangle\langle w n\rangle}
\end{aligned}
$$

after using the following identity

$$
\begin{aligned}
\langle 1 n\rangle^{2} & \left(-\langle n|N| 2]\langle n|N+2| 1]^{3}+\langle n|N+1| 2]\langle n|N| 1]^{3}\right) \\
+ & \langle 2 n\rangle^{2}\left(\langle n|N| 1]\langle n|N+1| 2]^{3}-\langle n|N+2| 1]\langle n|N| 2]^{3}\right) \\
& =-[12](\langle 1 n\rangle\langle n|N| 1]+\langle 2 n\rangle\langle n|N| 2])^{3}-[12]^{3}\langle 1 n\rangle^{2}\langle 2 n\rangle^{2}(\langle 1 n\rangle\langle n|N| 1]+\langle 2 n\rangle\langle n|N| 2]) \\
\quad & =-[12]\langle 2 n\rangle^{3}\langle n|N| \hat{2}]^{3}-[12]^{3}\langle 1 n\rangle^{2}\langle 2 n\rangle^{3}\langle n|N| \hat{2}] .
\end{aligned}
$$

Now note the second and third terms in (2.27) can be regrouped as

$$
-\frac{[12]^{3}\langle 1 n\rangle^{2}\langle 2 n\rangle^{3}}{\prod_{i \neq 1,2, n}\langle i 2\rangle}\left(\sum_{N}\langle 2|N| \hat{n}]\langle n|N| \hat{2}] \frac{\left|\psi_{N}\right|_{x}^{x}}{\langle x 2\rangle\langle x n\rangle} \frac{\left|\psi_{M}\right|_{y}^{y}}{\langle y 2\rangle\langle y n\rangle}+\frac{[\hat{2} \hat{n}]}{\langle 2 n\rangle} \frac{\left|\psi_{N \cup M}\right|_{w}^{w}}{\langle w 2\rangle\langle w n\rangle}\right)=0,
$$

which is exactly identity (1.4) for the set $\{\hat{2}, \ldots, \hat{n}\}$ ! Therefore we are left with

$$
\frac{\langle 21\rangle\langle 1 n\rangle}{\langle 2 n\rangle^{2}}\left(I_{1}+I_{2}\right) \rightarrow-\frac{[12]\langle 2 n\rangle^{3}}{\prod_{i \neq 1,2, n}\langle i 2\rangle} \sum_{N}\langle 2|N| \hat{n}]\langle n|N| \hat{2}]^{3} \frac{\left|\psi_{N}\right|_{x}^{x}}{\langle x 2\rangle\langle x n\rangle} \frac{\left|\psi_{M}\right|_{y}^{y}}{\langle y 2\rangle\langle y n\rangle} .
$$

To settle this leftover, we look back to $I_{b \neq 1,2, n}$ in (2.23) and find

$$
\frac{\left|\psi_{N_{b} \cup\{1,2\}}\right|_{1}^{1}}{\langle 1 b\rangle\langle 1 n\rangle} \rightarrow \frac{[21]}{\langle 21\rangle}\left|\psi_{N_{b} \cup\{\hat{2}\}}\right|_{\hat{2}}^{\hat{2}}=-\frac{[12]}{\langle 21\rangle}\langle 2 b\rangle\langle 2 n\rangle \frac{\left|\psi_{N_{b} \cup\{\hat{2}\}}\right|_{x}^{x}}{\langle x b\rangle\langle x n\rangle},
$$

where again we have used the independence of choice $x$ to switch the deleted row and column. Now

$$
\begin{aligned}
\frac{\langle 21\rangle\langle 1 n\rangle}{\langle 2 n\rangle^{2}} I_{b \neq 1,2, n} \rightarrow & -[12] \sum_{b \neq 1,2, n} \frac{\langle b n\rangle^{3}}{\prod_{i \neq 1, b, n}\langle i b\rangle} \\
& \times \sum_{N_{b}}\left(\left\langle b\left|N_{b}+\hat{2}\right| \hat{n}\right]\left\langle n\left|N_{b}+\hat{2}\right| b\right]^{3} \frac{\left|\psi_{N_{b} \cup\{\hat{2}\}}\right|_{x}^{x}}{\langle x b\rangle\langle x n\rangle} \frac{\left|\psi_{M_{b}}\right|_{y}^{y}}{\langle y b\rangle\langle y n\rangle}\right. \\
& \left.+\left\langle b\left|N_{b}\right| \hat{n}\right]\left\langle n\left|N_{b}\right| b\right]^{3} \frac{\left|\psi_{N_{b}}\right|_{x}^{x}}{\langle x b\rangle\langle x n\rangle} \frac{\left|\psi_{M_{b} \cup\{\hat{2}\}}\right|_{y}^{y}}{\langle y b\rangle\langle y n\rangle}\right) .
\end{aligned}
$$


Summing (2.30) and (2.32), we get

$$
\begin{aligned}
& -\frac{\langle 21\rangle\langle 1 n\rangle}{\langle 2 n\rangle^{2}[12]}\left(I_{1}+I_{2}+I_{b \neq 1,2, n}\right) \\
& \rightarrow \frac{\langle 2 n\rangle^{3}}{\prod_{i \neq 1,2, n}\langle i 2\rangle} \sum_{N}\langle 2|N| \hat{n}]\langle n|N| \hat{2}]^{3} \frac{\left|\psi_{N}\right|_{x}^{x}}{\langle x 2\rangle\langle x n\rangle} \frac{\left|\psi_{M}\right|_{y}^{y}}{\langle y 2\rangle\langle y n\rangle} \\
& \quad+\sum_{b \neq 1,2, n} \frac{\langle b n\rangle^{3}}{\prod_{i \neq 1, b, n}\langle i b\rangle} \sum_{N_{b}^{\prime}}\left\langle b\left|N_{b}^{\prime}\right| \hat{n}\right]\left\langle n\left|N_{b}^{\prime}\right| b\right]^{3} \frac{\left|\psi_{N_{b}^{\prime}}\right|_{x}^{x}}{\langle x b\rangle\langle x n\rangle} \frac{\left|\psi_{M_{b}^{\prime}}\right|_{y}^{y}}{\langle y b\rangle\langle y n\rangle} \\
& =\sum_{b \neq 1, n} \frac{\langle b n\rangle^{3}}{\prod_{i \neq 1, b, n}\langle i b\rangle} \sum_{N^{\prime}}\left\langle b\left|N^{\prime}\right| \hat{n}\right]\left\langle n\left|N^{\prime}\right| b\right]^{3} \frac{\left|\psi_{N^{\prime}}\right|_{x}^{x}}{\langle x b\rangle\langle x n\rangle} \frac{\left|\psi_{M^{\prime}}\right|_{y}^{y}}{\langle y b\rangle\langle y n\rangle}=0,
\end{aligned}
$$

which returns to the form of $(2.8)$ for the set $\{\hat{2}, \ldots, \hat{n}\}$ ! It vanishes after assuming the identity of $(n-1)$ particles (without particle 1) holds. Similar to $N^{\prime}, M^{\prime}$, here $N_{b}^{\prime}, M_{b}^{\prime}$ denote the sets including $\hat{2}$ but not $b$. This finishes the inductive proof of (1.3).

\section{Two identities of the KLT momentum kernel}

In this section we will prove (1.5) and (1.6) as conjectured in [14]. To understand these relations, we must first define the transformation matrices $D$ and $C$ between BCJ basis of gauge amplitudes via

$$
\begin{aligned}
& A_{n}\left(t, \alpha_{t}, n-1, n\right)=\sum_{\alpha_{t^{\prime}} \in S_{n-3}} A_{n}\left(t^{\prime}, \alpha_{t^{\prime}}, n-1, n\right) D\left[t^{\prime}, \alpha_{t^{\prime}}, n-1, n \mid t, \alpha_{t}, n-1, n\right], \\
& \widetilde{A}_{n}\left(t, n-1, \beta_{t}, n\right)=\sum_{\beta_{t^{\prime}} \in S_{n-3}} C\left[t, n-1, \beta_{t}, n \mid t^{\prime}, n-1, \beta_{t^{\prime}}, n\right] \widetilde{A}_{n}\left(t^{\prime}, n-1, \beta_{t^{\prime}}, n\right),
\end{aligned}
$$

where $\alpha_{t^{\prime}}$ and $\beta_{t^{\prime}}$ denote the permutations of $(n-3)$ particles other than $t^{\prime},(n-1)$ and $n$. In a tensorial sense, $D$ and $C$ are the transformation matrices with respect to the summation of all $(n-3)$ ! permutations, which is defined as the inner product. For reader's reference, we write (1.5) and (1.6) again below

$$
\begin{gathered}
\sum_{\alpha_{t^{\prime}}, \beta_{t^{\prime}} \in S_{n-3}} D\left[t, \alpha_{t}, n-1, n \mid t^{\prime}, \alpha_{t^{\prime}}, n-1, n\right] S\left[\alpha_{t^{\prime}} \mid \beta_{t^{\prime}}\right]_{p_{n-1}} \\
\cdot C\left[t^{\prime}, n-1, \beta_{t^{\prime}}, n \mid t, n-1, \beta_{t}, n\right]=S\left[\alpha_{t} \mid \beta_{t}\right] p_{n-1}, \\
\sum_{t^{\prime}=1}^{n-2} \sum_{\alpha_{t^{\prime}}, \beta_{t^{\prime}} \in S_{n-3}} D\left[t, \alpha_{t}, n-1, n \mid t^{\prime}, \alpha_{t^{\prime}}, n-1, n\right] S\left[\alpha_{t^{\prime}} \mid \beta_{t^{\prime}}\right]_{p_{n-1}} \\
\cdot J_{t^{\prime}}\left(C\left[t^{\prime}, n-1, \beta_{t^{\prime}}, n \mid t, n-1, \beta_{t}, n\right]\right)=0
\end{gathered}
$$

where $S\left[\alpha_{t} \mid \beta_{t}\right]_{p_{n-1}}$ is the KLT momentum kernel of pivot $p_{n-1}$, and $J_{t^{\prime}} \equiv J_{t^{\prime}, \dot{\alpha} \dot{\beta}}$ is the anti-holomorphic angular momentum operator. Here we follow the convention of $S$ in [1618], namely

$$
S\left[\alpha_{1}, \ldots, \alpha_{k} \mid \beta_{1}, \ldots, \beta_{k}\right]_{p_{n-1}}=\prod_{i=1}^{k}\left(s_{\alpha_{i}, n-1}+\sum_{j=i+1}^{k} \theta\left(\alpha_{i}, \alpha_{j}\right) s_{\alpha_{i}, \alpha_{j}}\right),
$$


where $s_{i j}$ is each Mandelstam variable, and $\theta\left(\alpha_{i}, \alpha_{j}\right)$ is zero when the pair $\left(\alpha_{i}, \alpha_{j}\right)$ has the same ordering at both sets $\left\{\alpha_{1}, \ldots, \alpha_{k}\right\}$ and $\left\{\beta_{1}, \ldots, \beta_{k}\right\}$, and unity otherwise.

For the first identity, its physical interpretation is straightforward: if we regard the KLT momentum kernel $S$ as the metric, it is simply the tensorial transformation rule for metric. In fact, such a tensorial formulation had been established in [20, 22] (known as the KLT orthogonality or the CHY formulation) and we will use it to formally prove the first identity shortly. The second identity is however more intricate, as it roughly represents angular momentum conservation in an entangled way. The CHY formulation can help transform it into a relation that may reveal very nontrivial properties of scattering process, while to prove it directly is yet beyond our understanding.

\subsection{Proof of the first identity}

Before the proof, we must first rewrite gauge amplitudes in the CHY formulation [22] which is based on the scattering equations [21]. It tells that

$$
\begin{aligned}
& A_{n}\left(t, \alpha_{t}, n-1, n\right)=\sum_{i=1}^{(n-3) !} \frac{1}{\operatorname{det}^{\prime}(\Phi)\left(\sigma^{(i)}\right)} \Sigma^{(i)}\left(t, \alpha_{t}, n-1, n\right) \operatorname{Pf}^{\prime} \Psi\left(\sigma^{(i)}\right), \\
& \widetilde{A}_{n}\left(t, n-1, \beta_{t}, n\right)=\sum_{i=1}^{(n-3) !} \frac{1}{\operatorname{det}^{\prime}(\Phi)\left(\sigma^{(i)}\right)} \Sigma^{(i)}\left(t, n-1, \beta_{t}, n\right) \operatorname{Pf}^{\prime} \Psi\left(\sigma^{(i)}\right),
\end{aligned}
$$

where $\sigma^{(i)}$ denotes the $i$-th solution to the scattering equations

$$
\sum_{b \neq a} \frac{s_{a b}}{\sigma_{a b}}=0
$$

with $\sigma_{a b}=\sigma_{a}-\sigma_{b}$, and there are $(n-3)$ ! solutions in total. The definitions of $\operatorname{det}^{\prime}(\Phi)$ and $\operatorname{Pf}^{\prime} \Psi$, namely the reduced determinant of Jacobian $\Phi$ and the reduced Pffafian of antisymmetric matrix $\Psi$, can be found in [22]. The object mainly concerns us is

$$
\Sigma^{(i)}(\alpha) \equiv \frac{1}{\sigma_{\alpha(1), \alpha(2)}^{(i)} \ldots \sigma_{\alpha(n-1), \alpha(n)}^{(i)} \sigma_{\alpha(n), \alpha(1)}^{(i)}} .
$$

On the other hand, the KLT relation gives

$$
\begin{aligned}
(-)^{n+1} M_{n}(1, \ldots, n) & =\sum_{\alpha_{t}, \beta_{t} \in S_{n-3}} A_{n}\left(t, \alpha_{t}, n-1, n\right) S\left[\alpha_{t} \mid \beta_{t}\right]_{p_{n-1}} \widetilde{A}_{n}\left(t, n-1, \beta_{t}, n\right) \\
& =\sum_{i=1}^{(n-3) !} \frac{\operatorname{Pf}^{\prime} \Psi\left(\sigma^{(i)}\right) \mathrm{Pf}^{\prime} \Psi\left(\sigma^{(i)}\right)}{\operatorname{det}^{\prime}(\Phi)\left(\sigma^{(i)}\right)}
\end{aligned}
$$

where the second line results from the CHY formulation. There is a subtle issue of the sign above, due to the different conventions $M_{n}=-M_{n}^{\mathrm{CHY}}$ and $S\left[\alpha_{t} \mid \beta_{t}\right]=S^{\mathrm{CHY}}\left[\beta_{t} \mid \alpha_{t}^{\mathrm{T}}\right]$. Plugging (3.6) and (3.7) into this relation, yields

$$
\sum_{\alpha_{t}, \beta_{t} \in S_{n-3}} \Sigma^{(i)}\left(t, \alpha_{t}, n-1, n\right) S\left[\alpha_{t} \mid \beta_{t}\right]_{p_{n-1}} \Sigma^{(j)}\left(t, n-1, \beta_{t}, n\right)=\operatorname{det}^{\prime}(\Phi)\left(\sigma^{(i)}\right) \delta_{i j},
$$


or more compactly,

$$
G_{i \alpha_{t}} S\left[\alpha_{t} \mid \beta_{t}\right]\left(H_{j \beta_{t}}\right)^{\mathrm{T}}=I_{(n-3) ! \times(n-3) !},
$$

which is the KLT orthogonality, if we define matrices

$$
G_{i \alpha_{t}} \equiv \frac{\Sigma^{(i)}\left(t, \alpha_{t}, n-1, n\right)}{\sqrt{\operatorname{det}^{\prime}(\Phi)\left(\sigma^{(i)}\right)}}, H_{j \beta_{t}} \equiv \frac{\Sigma^{(j)}\left(t, n-1, \beta_{t}, n\right)}{\sqrt{\operatorname{det}^{\prime}(\Phi)\left(\sigma^{(j)}\right)}} .
$$

From this matrix relation we immediately get

$$
S\left[\alpha_{t} \mid \beta_{t}\right]=\left(G_{i \alpha_{t}}\right)^{-1}\left(\left(H_{i \beta_{t}}\right)^{\mathrm{T}}\right)^{-1} .
$$

Back to (3.6) and (3.7), if we further define the row vector

$$
\Theta_{i} \equiv \frac{\operatorname{Pf}^{\prime} \Psi\left(\sigma^{(i)}\right)}{\sqrt{\operatorname{det}^{\prime}(\Phi)\left(\sigma^{(i)}\right)}}
$$

then

$$
A_{n}\left(t, \alpha_{t}, n-1, n\right)=\Theta_{i} G_{i \alpha_{t}}, \quad \widetilde{A}_{n}\left(t, n-1, \beta_{t}, n\right)=\Theta_{i} H_{i \beta_{t}} .
$$

Plugging them back into (3.1) and (3.2), and assuming their independence of basis $\Theta_{i}$, we get

$$
\begin{aligned}
G_{i \alpha_{t}} & =G_{i \alpha_{t^{\prime}}} D\left[t^{\prime}, \alpha_{t^{\prime}}, n-1, n \mid t, \alpha_{t}, n-1, n\right], \\
H_{i \beta_{t}} & =H_{i \beta_{t^{\prime}}}\left(C\left[t, n-1, \beta_{t}, n \mid t^{\prime}, n-1, \beta_{t^{\prime}}, n\right]\right)^{\mathrm{T}},
\end{aligned}
$$

or equivalently,

$$
\begin{aligned}
D\left[t^{\prime}, \alpha_{t^{\prime}}, n-1, n \mid t, \alpha_{t}, n-1, n\right] & =\left(G_{i \alpha_{t^{\prime}}}\right)^{-1} G_{i \alpha_{t}}, \\
C\left[t, n-1, \beta_{t}, n \mid t^{\prime}, n-1, \beta_{t^{\prime}}, n\right] & =\left(H_{i \beta_{t}}\right)^{\mathrm{T}}\left(\left(H_{i \beta_{t^{\prime}}}\right)^{-1}\right)^{\mathrm{T}} .
\end{aligned}
$$

Finally we plug them back into the l.h.s. of (1.5) and interchange $t$ and $t^{\prime}$, together with (3.14) we get

$$
\begin{gathered}
\sum_{\alpha_{t^{\prime}}, \beta_{t^{\prime}} \in S_{n-3}} D\left[t, \alpha_{t}, n-1, n \mid t^{\prime}, \alpha_{t^{\prime}}, n-1, n\right] S\left[\alpha_{t^{\prime}} \mid \beta_{t^{\prime}}\right]_{p_{n-1}} C\left[t^{\prime}, n-1, \beta_{t^{\prime}}, n \mid t, n-1, \beta_{t}, n\right] \\
=\left(G_{i \alpha_{t}}\right)^{-1} G_{i \alpha_{t^{\prime}}}\left(G_{j \alpha_{t^{\prime}}}\right)^{-1}\left(\left(H_{j \beta_{t^{\prime}}}\right)^{\mathrm{T}}\right)^{-1}\left(H_{k \beta_{t^{\prime}}}\right)^{\mathrm{T}}\left(\left(H_{k \beta_{t}}\right)^{-1}\right)^{\mathrm{T}} \\
=\left(G_{i \alpha_{t}}\right)^{-1}\left(\left(H_{i \beta_{t}}\right)^{\mathrm{T}}\right)^{-1}=S\left[\alpha_{t} \mid \beta_{t}\right]_{p_{n-1}},
\end{gathered}
$$

which is exactly the r.h.s. of (1.5), hence the proof is finished. 


\subsection{Discussion of the second identity}

Now we move to prove (1.6). Equipped with the matrices defined in the previous subsection, the 1.h.s. of (1.6) can be simplified as

$$
\begin{aligned}
& \sum_{t^{\prime}=1}^{n-2}\left(G_{i \alpha_{t}}\right)^{-1} G_{i \alpha_{t^{\prime}}}\left(G_{j \alpha_{t^{\prime}}}\right)^{-1}\left(\left(H_{j \beta_{t^{\prime}}}\right)^{\mathrm{T}}\right)^{-1} \cdot J_{t^{\prime}}\left[\left(H_{k \beta_{t^{\prime}}}\right)^{\mathrm{T}}\left(\left(H_{k \beta_{t}}\right)^{-1}\right)^{\mathrm{T}}\right] \\
& =\sum_{t^{\prime}=1}^{n-2}\left(G_{i \alpha_{t}}\right)^{-1}\left(\left(H_{i \beta_{t^{\prime}}}\right)^{\mathrm{T}}\right)^{-1} \cdot J_{t^{\prime}}\left[\left(H_{j \beta_{t^{\prime}}}\right)^{\mathrm{T}}\left(\left(H_{j \beta_{t}}\right)^{-1}\right)^{\mathrm{T}}\right] \\
& =\sum_{t^{\prime}=1}^{n-2}\left(G_{i \alpha_{t}}\right)^{-1}\left[\left(\left(H_{i \beta_{t^{\prime}}}\right)^{\mathrm{T}}\right)^{-1}\left(H_{j \beta_{t^{\prime}}}\right)^{\mathrm{T}} \cdot J_{t^{\prime}}\left(\left(H_{j \beta_{t}}\right)^{-1}\right)^{\mathrm{T}}\right. \\
& \left.\quad+\left(\left(H_{i \beta_{t^{\prime}}}\right)^{\mathrm{T}}\right)^{-1} \cdot J_{t^{\prime}}\left(H_{j \beta_{t^{\prime}}}\right)^{\mathrm{T}} \cdot\left(\left(H_{j \beta_{t}}\right)^{-1}\right)^{\mathrm{T}}\right] \\
& =\sum_{t^{\prime}=1}^{n-2}\left(G_{i \alpha_{t}}\right)^{-1}\left[J_{t^{\prime}}\left(\left(H_{i \beta_{t}}\right)^{-1}\right)^{\mathrm{T}} \cdot\left(H_{j \beta_{t}}\right)^{\mathrm{T}}+\left(\left(H_{i \beta_{t^{\prime}}}\right)^{\mathrm{T}}\right)^{-1} \cdot J_{t^{\prime}}\left(H_{j \beta_{t^{\prime}}}\right)^{\mathrm{T}}\right]\left(\left(H_{j \beta_{t}}\right)^{-1}\right)^{\mathrm{T}},
\end{aligned}
$$

assuming the two matrices in the front and end of the last line are non-degenerate, we should prove

$$
\sum_{t^{\prime}=1}^{n-2}\left[J_{t^{\prime}}\left(\left(H_{i \beta_{t}}\right)^{-1}\right)^{\mathrm{T}} \cdot\left(H_{j \beta_{t}}\right)^{\mathrm{T}}+\left(\left(H_{i \beta_{t^{\prime}}}\right)^{\mathrm{T}}\right)^{-1} \cdot J_{t^{\prime}}\left(H_{j \beta_{t^{\prime}}}\right)^{\mathrm{T}}\right]=0 .
$$

For the first term above, the summation over $t^{\prime}$ is trivial since the matrix product involves $t$ only, so it is in fact

$$
\left(\sum_{t^{\prime}=1}^{n-2} J_{t^{\prime}}\right)\left(\left(H_{i \beta_{t}}\right)^{-1}\right)^{\mathrm{T}} \cdot\left(H_{j \beta_{t}}\right)^{\mathrm{T}}=0
$$

due to angular momentum conservation, as the absence of $J_{n-1}$ and $J_{n}$ does not matter since $\tilde{\lambda}_{n-1}$ and $\tilde{\lambda}_{n}$ have been solved by momentum conservation (see [14] for more details). Therefore we are left with

$$
\sum_{t=1}^{n-2}\left(\left(H_{i \beta_{t}}\right)^{\mathrm{T}}\right)^{-1} \cdot J_{t}\left(H_{j \beta_{t}}\right)^{\mathrm{T}}=0
$$

where the dummy variable $t^{\prime}$ has been replaced by $t$. We can continue to transform it into a convenient form for further attempts to prove, by isolating its real matrix content. Let's define

$$
\Sigma_{j \beta_{t}} \equiv \Sigma^{(j)}\left(t, n-1, \beta_{t}, n\right), \quad W_{i j} \equiv \frac{1}{\sqrt{\operatorname{det}^{\prime}(\Phi)\left(\sigma^{(i)}\right)}} \delta_{i j}
$$


then it is clear that $H_{i \beta_{t}}=W_{i j} \Sigma_{j \beta_{t}}$. While $W_{i j}$ is a trivial diagonal matrix, $\Sigma_{j \beta_{t}}$ encodes the real matrix content. Now we can write the l.h.s. of (3.23) as

$$
\begin{aligned}
\sum_{t=1}^{n-2} W^{-1} & \left(\left(\Sigma_{i \beta_{t}}\right)^{\mathrm{T}}\right)^{-1} \cdot J_{t}\left(\left(\Sigma_{j \beta_{t}}\right)^{\mathrm{T}} W\right) \\
& =\sum_{t=1}^{n-2} W^{-1}\left(\left(\Sigma_{i \beta_{t}}\right)^{\mathrm{T}}\right)^{-1} \cdot J_{t}\left(\Sigma_{j \beta_{t}}\right)^{\mathrm{T}} \cdot W+\sum_{t=1}^{n-2} W^{-1} \cdot J_{t} W \\
& =\sum_{t=1}^{n-2}\left(\left(\Sigma_{i \beta_{t}}\right)^{\mathrm{T}}\right)^{-1} \cdot J_{t}\left(\Sigma_{j \beta_{t}}\right)^{\mathrm{T}}+W^{-1} \cdot\left(\sum_{t=1}^{n-2} J_{t}\right) W \\
& =\sum_{t=1}^{n-2}\left(\left(\Sigma_{i \beta_{t}}\right)^{\mathrm{T}}\right)^{-1} \cdot J_{t}\left(\Sigma_{j \beta_{t}}\right)^{\mathrm{T}}
\end{aligned}
$$

where in the third line, the second term vanishes again due to angular momentum conservation. Finally, we are left with

$$
\sum_{t=1}^{n-2}\left(\left(\Sigma_{i \beta_{t}}\right)^{\mathrm{T}}\right)^{-1} \cdot J_{t}\left(\Sigma_{j \beta_{t}}\right)^{\mathrm{T}}=0,
$$

which can no longer be further simplified.

To get some sense of this very nontrivial identity, it is helpful to see the first nontrivial case $n=4$, which corresponds to the first nonempty $\beta_{t}$. Recall that

$$
\Sigma^{(i)}(\alpha)=\frac{1}{\sigma_{\alpha(1), \alpha(2)}^{(i)} \ldots \sigma_{\alpha(n-1), \alpha(n)}^{(i)} \sigma_{\alpha(n), \alpha(1)}^{(i)}},
$$

we have

$$
\begin{aligned}
& \left(\Sigma^{(i)}(1,3,2,4)\right)^{-1} J_{1} \Sigma^{(j)}(1,3,2,4)+\left(\Sigma^{(i)}(2,3,1,4)\right)^{-1} J_{2} \Sigma^{(j)}(2,3,1,4) \\
& \quad=\sigma_{13}^{(i)} \sigma_{32}^{(i)} \sigma_{24}^{(i)} \sigma_{41}^{(i)} J_{1}\left(\frac{1}{\sigma_{13}^{(j)} \sigma_{32}^{(j)} \sigma_{24}^{(j)} \sigma_{41}^{(j)}}\right)+\sigma_{23}^{(i)} \sigma_{31}^{(i)} \sigma_{14}^{(i)} \sigma_{42}^{(i)} J_{2}\left(\frac{1}{\sigma_{23}^{(j)} \sigma_{31}^{(j)} \sigma_{14}^{(j)} \sigma_{42}^{(j)}}\right) \\
& =\sigma_{13}^{(i)} \sigma_{32}^{(i)} \sigma_{24}^{(i)} \sigma_{41}^{(i)}\left(J_{1}+J_{2}\right)\left(\frac{1}{\sigma_{13}^{(j)} \sigma_{32}^{(j)} \sigma_{24}^{(j)} \sigma_{41}^{(j)}}\right)=0
\end{aligned}
$$

which trivially holds by the antisymmetry of $\sigma_{a b}$ ! But as $n$ increases, even for $n=5$ this identity will be much more entangled and simple antisymmetry is insufficient for its proof. The potential toolkit for this purpose includes: (1) relations of spinor derivatives on scattering equations; (2) KK and BCJ relations of $\Sigma_{j \beta_{t}}$; (3) induction, which may involve contour integration. We will come back to this point in the future after better understanding the scattering equations and their solutions.

A last comment is that in (1.6), the anti-holomorphic angular momentum operator $J_{t^{\prime}, \dot{\alpha} \dot{\beta}}$ should be generalized to $J_{t^{\prime}, \mu \nu}$ in arbitrary dimensions. Since in 4-dimension $J_{\mu \nu} \sim \varepsilon_{\alpha \beta} J_{\dot{\alpha} \dot{\beta}}+\varepsilon_{\dot{\alpha} \dot{\beta}} J_{\alpha \beta}$, and the soft theorem must hold for both holomorphic and antiholomorphic soft limits, it is more natural to use $J_{\mu \nu}$ as all other quantities are already defined for arbitrary dimensions. 


\section{Acknowledgments}

The authors would like to thank Qingjun Jin for reading the manuscript. This work is supported by Qiu-Shi Funding and Chinese NSF funding under contracts No.11135006, No.11125523 and No.11575156.

Open Access. This article is distributed under the terms of the Creative Commons Attribution License (CC-BY 4.0), which permits any use, distribution and reproduction in any medium, provided the original author(s) and source are credited.

\section{References}

[1] F.E. Low, Scattering of light of very low frequency by systems of spin 1/2, Phys. Rev. 96 (1954) 1428 [INSPIRE].

[2] M. Gell-Mann and M.L. Goldberger, Scattering of low-energy photons by particles of spin 1/2, Phys. Rev. 96 (1954) 1433 [InSPIRE].

[3] S. Saito, Low-energy theorem for Compton scattering, Phys. Rev. 184 (1969) 1894 [INSPIRE].

[4] F.E. Low, Bremsstrahlung of very low-energy quanta in elementary particle collisions, Phys. Rev. 110 (1958) 974 [INSPIRE].

[5] S. Weinberg, Photons and gravitons in s matrix theory: derivation of charge conservation and equality of gravitational and inertial mass, Phys. Rev. 135 (1964) B1049 [INSPIRE].

[6] S. Weinberg, Infrared photons and gravitons, Phys. Rev. 140 (1965) B516 [InSPIRE].

[7] F. Cachazo and A. Strominger, Evidence for a new soft graviton theorem, arXiv:1404.4091 [INSPIRE].

[8] R. Britto, F. Cachazo and B. Feng, New recursion relations for tree amplitudes of gluons, Nucl. Phys. B 715 (2005) 499 [hep-th/0412308] [inSPIRE].

[9] R. Britto, F. Cachazo, B. Feng and E. Witten, Direct proof of tree-level recursion relation in Yang-Mills theory, Phys. Rev. Lett. 94 (2005) 181602 [hep-th/0501052] [INSPIRE].

[10] E. Casali, Soft sub-leading divergences in Yang-Mills amplitudes, JHEP 08 (2014) 077 [arXiv: 1404.5551] [INSPIRE].

[11] S. He, Y.-t. Huang and C. Wen, Loop corrections to soft theorems in gauge theories and gravity, JHEP 12 (2014) 115 [arXiv: 1405.1410] [INSPIRE].

[12] Z. Bern, L.J. Dixon, M. Perelstein and J.S. Rozowsky, Multileg one loop gravity amplitudes from gauge theory, Nucl. Phys. B 546 (1999) 423 [hep-th/9811140] [INSPIRE].

[13] B. Feng and S. He, Graphs, determinants and gravity amplitudes, JHEP 10 (2012) 121 [arXiv:1207.3220] [INSPIRE].

[14] Y.-J. Du, B. Feng, C.-H. Fu and Y. Wang, Note on soft graviton theorem by KLT relation, JHEP 11 (2014) 090 [arXiv: 1408.4179] [INSPIRE].

[15] H. Kawai, D.C. Lewellen and S.H.H. Tye, A relation between tree amplitudes of closed and open strings, Nucl. Phys. B 269 (1986) 1 [INSPIRE].

[16] N.E.J. Bjerrum-Bohr, P.H. Damgaard, B. Feng and T. Sondergaard, Gravity and Yang-Mills amplitude relations, Phys. Rev. D 82 (2010) 107702 [arXiv:1005.4367] [INSPIRE]. 
[17] N.E.J. Bjerrum-Bohr, P.H. Damgaard, B. Feng and T. Sondergaard, New identities among gauge theory amplitudes, Phys. Lett. B 691 (2010) 268 [arXiv:1006.3214] [INSPIRE].

[18] N.E.J. Bjerrum-Bohr, P.H. Damgaard, B. Feng and T. Sondergaard, Proof of gravity and Yang-Mills amplitude relations, JHEP 09 (2010) 067 [arXiv: 1007.3111] [INSPIRE].

[19] Z. Bern, J.J.M. Carrasco and H. Johansson, New relations for gauge-theory amplitudes, Phys. Rev. D 78 (2008) 085011 [arXiv: 0805.3993] [INSPIRE].

[20] F. Cachazo, S. He and E.Y. Yuan, Scattering equations and Kawai-Lewellen-Tye orthogonality, Phys. Rev. D 90 (2014) 065001 [arXiv:1306.6575] [InSPIRE].

[21] F. Cachazo, S. He and E.Y. Yuan, Scattering of massless particles in arbitrary dimensions, Phys. Rev. Lett. 113 (2014) 171601 [arXiv:1307.2199] [inSPIRE].

[22] F. Cachazo, S. He and E.Y. Yuan, Scattering of massless particles: scalars, gluons and gravitons, JHEP 07 (2014) 033 [arXiv:1309.0885] [INSPIRE].

[23] B.U.W. Schwab and A. Volovich, Subleading soft theorem in arbitrary dimensions from scattering equations, Phys. Rev. Lett. 113 (2014) 101601 [arXiv:1404.7749] [INSPIRE].

[24] N. Afkhami-Jeddi, Soft graviton theorem in arbitrary dimensions, arXiv:1405.3533 [INSPIRE].

[25] M. Zlotnikov, Sub-sub-leading soft-graviton theorem in arbitrary dimension, JHEP 10 (2014) 148 [arXiv: 1407.5936] [INSPIRE].

[26] C. Kalousios and F. Rojas, Next to subleading soft-graviton theorem in arbitrary dimensions, JHEP 01 (2015) 107 [arXiv: 1407.5982] [INSPIRE].

[27] B.U.W. Schwab, Subleading soft factor for string disk amplitudes, JHEP 08 (2014) 062 [arXiv: 1406.4172] [INSPIRE].

[28] M. Bianchi, S. He, Y.-t. Huang and C. Wen, More on soft theorems: trees, loops and strings, Phys. Rev. D 92 (2015) 065022 [arXiv: 1406.5155] [INSPIRE].

[29] B.U.W. Schwab, A note on soft factors for closed string scattering, JHEP 03 (2015) 140 [arXiv:1411.6661] [INSPIRE].

[30] W.-M. Chen, Y.-t. Huang and C. Wen, New fermionic soft theorems for supergravity amplitudes, Phys. Rev. Lett. 115 (2015) 021603 [arXiv:1412.1809] [INSPIRE].

[31] P. Di Vecchia, R. Marotta and M. Mojaza, Soft theorem for the graviton, dilaton and the Kalb-Ramond field in the bosonic string, JHEP 05 (2015) 137 [arXiv: 1502. 05258] [INSPIRE].

[32] M. Bianchi and A.L. Guerrieri, On the soft limit of open string disk amplitudes with massive states, JHEP 09 (2015) 164 [arXiv: 1505.05854] [INSPIRE].

[33] L.V. Bork and A.I. Onishchenko, On soft theorems and form factors in $\mathcal{N}=4 S Y M$ theory, JHEP 12 (2015) 030 [arXiv: 1506.07551] [INSPIRE].

[34] S. Chin, S. Lee and Y. Yun, ABJM amplitudes in U-gauge and a soft theorem, JHEP 11 (2015) 088 [arXiv: 1508.07975] [INSPIRE].

[35] M. Campiglia and A. Laddha, Asymptotic symmetries of gravity and soft theorems for massive particles, JHEP 12 (2015) 094 [arXiv: 1509. 01406] [INSPIRE].

[36] M. Bianchi and A.L. Guerrieri, On the soft limit of closed string amplitudes with massive states, Nucl. Phys. B 905 (2016) 188 [arXiv:1512.00803] [INSPIRE]. 
[37] A.J. Larkoski, Conformal invariance of the subleading soft theorem in gauge theory, Phys. Rev. D 90 (2014) 087701 [arXiv:1405.2346] [INSPIRE].

[38] T. Adamo, E. Casali and D. Skinner, Perturbative gravity at null infinity, Class. Quant. Grav. 31 (2014) 225008 [arXiv:1405.5122] [INSPIRE].

[39] Y. Geyer, A.E. Lipstein and L. Mason, Ambitwistor strings at null infinity and (subleading) soft limits, Class. Quant. Grav. 32 (2015) 055003 [arXiv:1406.1462] [INSPIRE].

[40] D. Kapec, V. Lysov, S. Pasterski and A. Strominger, Semiclassical Virasoro symmetry of the quantum gravity $\mathcal{S}$-matrix, JHEP 08 (2014) 058 [arXiv: 1406.3312] [INSPIRE].

[41] J. Broedel, M. de Leeuw, J. Plefka and M. Rosso, Constraining subleading soft gluon and graviton theorems, Phys. Rev. D 90 (2014) 065024 [arXiv:1406.6574] [INSPIRE].

[42] Z. Bern, S. Davies, P. Di Vecchia and J. Nohle, Low-energy behavior of gluons and gravitons from gauge invariance, Phys. Rev. D 90 (2014) 084035 [arXiv:1406.6987] [InSPIRE].

[43] C.D. White, Diagrammatic insights into next-to-soft corrections, Phys. Lett. B 737 (2014) 216 [arXiv: 1406.7184] [INSPIRE].

[44] T. He, P. Mitra, A.P. Porfyriadis and A. Strominger, New symmetries of massless QED, JHEP 10 (2014) 112 [arXiv:1407.3789] [INSPIRE].

[45] V. Lysov, S. Pasterski and A. Strominger, Low's subleading soft theorem as a symmetry of QED, Phys. Rev. Lett. 113 (2014) 111601 [arXiv:1407.3814] [INSPIRE].

[46] M. Campiglia and A. Laddha, Asymptotic symmetries and subleading soft graviton theorem, Phys. Rev. D 90 (2014) 124028 [arXiv:1408.2228] [INSPIRE].

[47] Z.-W. Liu, Soft theorems in maximally supersymmetric theories, Eur. Phys. J. C 75 (2015) 105 [arXiv: 1410.1616] [INSPIRE].

[48] J. Rao, Soft theorem of $\mathcal{N}=4$ SYM in grassmannian formulation, JHEP 02 (2015) 087 [arXiv: 1410.5047] [INSPIRE].

[49] D. Kapec, V. Lysov and A. Strominger, Asymptotic symmetries of massless QED in even dimensions, arXiv:1412.2763 [INSPIRE].

[50] A. Sabio Vera and M.A. Vazquez-Mozo, The double copy structure of soft gravitons, JHEP 03 (2015) 070 [arXiv: 1412.3699] [inSPIRE].

[51] A. Mohd, A note on asymptotic symmetries and soft-photon theorem, JHEP 02 (2015) 060 [arXiv: 1412.5365] [INSPIRE].

[52] H. Lüo, P. Mastrolia and W.J. Torres Bobadilla, Subleading soft behavior of QCD amplitudes, Phys. Rev. D 91 (2015) 065018 [arXiv:1411.1669] [INSPIRE].

[53] M. Campiglia and A. Laddha, New symmetries for the gravitational S-matrix, JHEP 04 (2015) 076 [arXiv: 1502.02318] [INSPIRE].

[54] S. Pasterski, A. Strominger and A. Zhiboedov, New gravitational memories, arXiv: 1502.06120 [INSPIRE].

[55] A.E. Lipstein, Soft theorems from conformal field theory, JHEP 06 (2015) 166 [arXiv: 1504.01364] [INSPIRE].

[56] D. Kapec, M. Pate and A. Strominger, New symmetries of QED, arXiv:1506.02906 [INSPIRE]. 
[57] S.G. Avery and B.U.W. Schwab, Burg-Metzner-Sachs symmetry, string theory and soft theorems, Phys. Rev. D 93 (2016) 026003 [arXiv: 1506.05789] [inSPIRE].

[58] T.T. Dumitrescu, T. He, P. Mitra and A. Strominger, Infinite-dimensional fermionic symmetry in supersymmetric gauge theories, arXiv:1511.07429 [INSPIRE].

[59] Z. Bern, S. Davies and J. Nohle, On loop corrections to subleading soft behavior of gluons and gravitons, Phys. Rev. D 90 (2014) 085015 [arXiv:1405.1015] [INSPIRE].

[60] F. Cachazo and E.Y. Yuan, Are soft theorems renormalized?, arXiv:1405.3413 [INSPIRE].

[61] A. Brandhuber, E. Hughes, B. Spence and G. Travaglini, One-loop soft theorems via dual superconformal symmetry, JHEP 03 (2016) 084 [arXiv: 1511.06716] [INSPIRE].

[62] D. Bonocore, E. Laenen, L. Magnea, L. Vernazza and C.D. White, The method of regions and next-to-soft corrections in Drell-Yan production, Phys. Lett. B 742 (2015) 375 [arXiv: 1410.6406] [INSPIRE].

[63] D. Bonocore, E. Laenen, L. Magnea, S. Melville, L. Vernazza and C.D. White, $A$ factorization approach to next-to-leading-power threshold logarithms, JHEP 06 (2015) 008 [arXiv: 1503.05156] [INSPIRE].

[64] W.-M. Chen, Y.-t. Huang and C. Wen, From U(1) to E 8 $_{8}$ soft theorems in supergravity amplitudes, JHEP 03 (2015) 150 [arXiv:1412.1811] [INSPIRE].

[65] F. Cachazo, S. He and E.Y. Yuan, New double soft emission theorems, Phys. Rev. D 92 (2015) 065030 [arXiv: 1503.04816] [INSPIRE].

[66] T. Klose, T. McLoughlin, D. Nandan, J. Plefka and G. Travaglini, Double-soft limits of gluons and gravitons, JHEP 07 (2015) 135 [arXiv: 1504.05558] [INSPIRE].

[67] A. Volovich, C. Wen and M. Zlotnikov, Double soft theorems in gauge and string theories, JHEP 07 (2015) 095 [arXiv: 1504.05559] [INSPIRE].

[68] Y.-J. Du and H. Lüo, On single and double soft behaviors in NLSM, JHEP 08 (2015) 058 [arXiv: 1505.04411] [INSPIRE].

[69] G. Georgiou, Multi-soft theorems in gauge theory from MHV diagrams, JHEP 08 (2015) 128 [arXiv: 1505.08130] [INSPIRE].

[70] P. Di Vecchia, R. Marotta and M. Mojaza, Double-soft behavior for scalars and gluons from string theory, JHEP 12 (2015) 150 [arXiv:1507.00938] [INSPIRE].

[71] I. Low, Double soft theorems and shift symmetry in nonlinear $\sigma$-models, Phys. Rev. D 93 (2016) 045032 [arXiv:1512.01232] [INSPIRE]. 